PROCEEDINGS OF THE

AMERICAN MATHEMATICAL SOCIETY

Volume 130, Number 9, Pages 2631-2639

S 0002-9939(02)06382-7

Article electronically published on February 12, 2002

\title{
REDUCING SUBSPACES OF WEIGHTED SHIFT OPERATORS
}

\author{
MICHAEL STESSIN AND KEHE ZHU
}

(Communicated by Joseph A. Ball)

\begin{abstract}
A complete description of the reducing subspaces of weighted unilateral shift operators of finite multiplicity is obtained.
\end{abstract}

\section{INTRODUCTION}

Let $T$ be a bounded linear operator on a Hilbert space $H$ and let $X$ be a closed subspace of $H$. Recall that $X$ is invariant under $T$, or $X$ is an invariant subspace of $T$, if $T$ maps $X$ into itself. We say that $X$ is a reducing subspace of $T$ if $X$ is invariant under both $T$ and its adjoint $T^{*}$. An operator $T$ on $H$ is irreducible if the only reducing subspaces of $T$ are $H$ and $\{0\}$.

The purpose of this paper is to give a complete description of the reducing subspaces of weighted unilateral shift operators of finite multiplicity. As usual we will realize these shift operators as multiplication by $z^{N}$ on certain Hilbert spaces of analytic functions on the unit disk.

Shift operators have been studied very extensively, and the related bibliography is quite elaborate. Here we mention two excellent surveys [5] and [6], which contain many results and references related to the topic of this paper.

Let $\omega=\left\{\omega_{0}, \omega_{1}, \cdots, \omega_{n}, \cdots\right\}$ be a sequence of positive numbers. We consider the Hilbert space $H_{\omega}^{2}$ consisting of analytic functions

$$
f(z)=\sum_{k=0}^{\infty} a_{k} z^{k}
$$

in the unit disk $\mathbb{D}$ such that

$$
\|f\|^{2}=\|f\|_{\omega}^{2}=\sum_{k=0}^{\infty} \omega_{k}\left|a_{k}\right|^{2}<\infty .
$$

It is well known that $M_{z}$, the operator of multiplication by $z$, is bounded on $H_{\omega}^{2}$ if and only if

$$
M=\sup \left\{\frac{\omega_{n+1}}{\omega_{n}}: n \geq 0\right\}<\infty
$$

(see Proposition 7 in [6]). Throughout the paper we fix a weight sequence $\omega$ satisfying this condition. In particular, $\omega_{n} \leq \omega_{0} M^{n}$ for all $n$.

It is also well known that $M_{z}$ is irreducible, namely, the operator $M_{z}$ has no proper reducing subspaces in $H_{\omega}^{2}$ (see Corollary 2 to Theorem 3 in [6]; we also

Received by the editors February 6, 2001 and, in revised form, April 1, 2001.

2000 Mathematics Subject Classification. Primary 47B37, 47A15. 
obtain this as Corollary 8 in Section 3). Throughout the paper we fix an integer $N>1$ and study the reducing subspace lattice of the operator

$$
S=M_{z}^{N}=M_{z^{N}}
$$

Note that $S$ is a weighted unilateral shift operator of multiplicity $N$.

It is clear that $S$ has proper reducing subspaces. More specifically, for each integer $n$ with $0 \leq n \leq N-1$, we let

$$
X_{n}=\operatorname{Span}\left\{z^{n+k N}: k=0,1,2, \cdots\right\}
$$

here and throughout the paper we use Span to denote the closed linear span of a set in a Hilbert space. Then each $X_{n}$ is obviously a reducing subspace of $S$. Surprisingly, for most weight sequences $\omega$, these are more or less the only reducing subspaces. To state our main results, we need to introduce more notions about reducing subspaces and weight sequences.

A reducing subspace $X$ of $S$ in $H_{\omega}^{2}$ will be called minimal if the only reducing subspaces of $S$ contained in $X$ are $X$ and $\{0\}$.

A weight sequence $\omega$ is of type I if for each $0 \leq n \leq N-1$ and $0 \leq m \leq N-1$ with $n \neq m$ there exists some integer $k>0$ such that

$$
\frac{\omega_{n+k N}}{\omega_{n}} \neq \frac{\omega_{m+k N}}{\omega_{m}}
$$

A weight sequence $\omega$ is of type II if it is not of type I. Thus $\omega$ is of type II if and only if there exist $0 \leq n \leq N-1$ and $0 \leq m \leq N-1$ with $n \neq m$ such that

$$
\frac{\omega_{n+k N}}{\omega_{n}}=\frac{\omega_{m+k N}}{\omega_{m}}
$$

for all integers $k>0$.

We can now state our main results.

Theorem A. Every reducing subspace $X$ of $S$ in $H_{\omega}^{2}$ contains a minimal reducing subspace. The reducing subspaces $X_{n}, 0 \leq n \leq N-1$, are all minimal. And every minimal reducing subspace of $S$ in $H_{\omega}^{2}$ is singly generated by a polynomial of degree less than $N$.

Theorem B. If $\omega$ is of type I, then $X_{n}, 0 \leq n \leq N-1$, are the only minimal reducing subspaces of $S$ in $H_{\omega}^{2}$. Moreover, $S$ has exactly $2^{N}$ distinct reducing subspaces in $H_{\omega}^{2}$ (counting the two trivial ones: $H_{\omega}^{2}$ and $\{0\}$ ).

Theorem C. If $\omega$ is of type II, then $S$ has minimal reducing subspaces other than the $X_{n}$ 's. In fact, $S$ has infinitely many distinct minimal reducing subspaces in $H_{\omega}^{2}$.

Theorem D. Every reducing subspace of $S$ in $H_{\omega}^{2}$ is a direct (orthogonal) sum of no more than $N$ minimal reducing subspaces.

A special case of our results was obtained in [7]. The structure of the reducing subspace lattice for unweighted unilateral shifts was described in [3] and [4]. We also mention that the papers [1] and [2] considered reducing subspaces for analytic Toeplitz operators on the Hardy space with symbols more general than inner functions. 


\section{TRANSPARENT POLYNOMIALS}

Recall that $N$ and $\omega$ are fixed throughout the paper. We now introduce a class of polynomials based on $N$ and $\omega$.

Suppose $0 \leq m \leq N-1$ and $0 \leq n \leq N-1$. We say that $m$ and $n$ are $(\omega-N)$-related if

$$
\frac{\omega_{n+k N}}{\omega_{n}}=\frac{\omega_{m+k N}}{\omega_{m}}
$$

for all positive integers $k$. In this case we write $m \sim n$.

Suppose $p$ is a polynomial of degree less than $N$,

$$
p(z)=\sum_{k=0}^{N-1} a_{k} z^{k} .
$$

We say that $p$ is transparent if for any two nonzero coefficients $a_{i}$ and $a_{j}$ of $p$ we have $i \sim j$. It is clear that every monomial is transparent. The following lemma shows that other transparent polynomials exist if and only if $i \sim j$ for some $0 \leq i \leq N-1$ and $0 \leq j \leq N-1$ with $i \neq j$.

Lemma 1. If we use the equivalence relation $\sim$ above to partition the set

$$
\{0,1, \cdots, N-1\}
$$

into equivalence classes $\Omega_{1}, \cdots, \Omega_{K}$, then for each $1 \leq k \leq K$, polynomials of the form

$$
p(z)=\sum\left\{a_{i} z^{i}: i \in \Omega_{k}\right\}
$$

are transparent.

Proof. This is obvious.

Given any nonzero polynomial $p$ of degree less than $N$,

$$
p(z)=a_{0}+a_{1} z+\cdots+a_{N-1} z^{N-1},
$$

we define polynomials $q_{1}, \cdots, q_{K}$ as follows:

$$
q_{k}(z)=\sum\left\{a_{i} z^{i}: i \in \Omega_{k}\right\}, \quad 1 \leq k \leq K .
$$

We then drop the zero polynomials in the sequence $\left\{q_{1}, \cdots, q_{K}\right\}$ and list the remaining ones as $\left\{p_{1}, \cdots, p_{n}\right\}$ in such a way that the order of zero at the origin of the polynomials $p_{k}$ is increasing in $k$. The resulting decomposition

$$
p=p_{1}+\cdots+p_{n}
$$

is then called the canonical decomposition of $p$.

Lemma 2. For any nonnegative integers $k$ and $n$ we have

$$
\left(S^{k}\right)^{*} S^{k}\left(z^{n}\right)=\frac{\omega_{n+k N}}{\omega_{n}} z^{n} .
$$

Proof. This follows from an easy calculation using the standard basis of $H_{\omega}^{2}$.

Lemma 3. If

$$
p(z)=\sum_{i=m}^{N-1} a_{i} z^{i}
$$


is a transparent polynomial, where $0 \leq m \leq N-1$ and $a_{m} \neq 0$, then

$$
\left(S^{k}\right)^{*} S^{k}(p)=\frac{\omega_{m+k N}}{\omega_{m}} p
$$

for every nonnegative integer $k$.

Proof. If $m \leq i \leq N-1$ and $a_{i} \neq 0$, then

$$
\left(S^{k}\right)^{*} S^{k}\left(z^{i}\right)=\frac{\omega_{i+k N}}{\omega_{i}} z^{i}=\frac{\omega_{m+k N}}{\omega_{m}} z^{i} .
$$

This clearly implies the desired result.

Let $\mathbb{S}$ be the vector space consisting of all finite linear combinations of finite products of the operators $S$ and $S^{*}$. For any nonzero function $f \in H_{\omega}^{2}$ the closure of $\mathbb{S} f=\{T f: T \in \mathbb{S}\}$ in $H_{\omega}^{2}$ is clearly a reducing subspace of $S$ (possibly the whole space) and will be denoted by $X_{f}$. We call $X_{f}$ the reducing subspace generated by $f$. It is clear that $X_{f}$ is the smallest reducing subspace containing $f$.

Lemma 4. If $f$ is a transparent polynomial, then

$$
X_{f}=\operatorname{Span}\left\{f z^{k N}: k=0,1,2, \cdots\right\} .
$$

Proof. Let

$$
X=\operatorname{Span}\left\{f z^{k N}: k=0,1,2, \cdots\right\} .
$$

Then $f \in X \subset X_{f}$. Since $X_{f}$ is the smallest reducing subspace of $S$ containing $f$, it suffices for us to show that $X$ is a reducing subspace of $S$ in $H_{\omega}^{2}$.

It is clear that $X$ is invariant under $S$. To show that $X$ is also invariant under $S^{*}$, we fix some positive integer $k$ and write $k=k^{\prime}+1$. Then

$$
S^{*}\left(f z^{k N}\right)=S^{*} S\left(f z^{k^{\prime} N}\right) .
$$

If

$$
f(z)=\sum_{i=m}^{N-1} a_{i} z^{i}
$$

where $a_{m} \neq 0$, then by Lemma 2

$$
S^{*}\left(f z^{k N}\right)=\sum_{i=m}^{N-1} a_{i} \frac{\omega_{i+k N}}{\omega_{i+k^{\prime} N}} z^{i+k^{\prime} N} .
$$

For each $i$ with $a_{i} \neq 0$, we have

$$
\frac{\omega_{i+k N}}{\omega_{i+k^{\prime} N}}=\frac{\omega_{i+k N}}{\omega_{i}} \cdot \frac{\omega_{i}}{\omega_{i+k^{\prime} N}}=\frac{\omega_{m+k N}}{\omega_{m}} \cdot \frac{\omega_{m}}{\omega_{m+k^{\prime} N}}=\frac{\omega_{m+k N}}{\omega_{m+k^{\prime} N}} .
$$

It follows that

$$
S^{*}\left(f z^{k N}\right)=\frac{\omega_{m+k N}}{\omega_{m+k^{\prime} N}} f z^{k^{\prime} N} .
$$

This shows that $X$ is invariant under $S^{*}$.

Lemma 5. Suppose $X$ is a reducing subspace of $S$ in $H_{\omega}^{2}$ and

$$
p=p_{1}+p_{2}+\cdots+p_{n}
$$

is the canonical decomposition of a polynomial of degree less than $N$ into transparent polynomials. If $p \in X$, then $p_{i} \in X$ for each $i=1,2, \cdots, n$. 
Proof. For each $1 \leq i \leq n$ write

$$
p_{i}(z)=\sum_{j=m_{i}}^{N-1} a_{j}^{(i)} z^{j}, \quad a_{m_{i}}^{(i)} \neq 0 .
$$

Note that the $p_{i}$ 's are mutually orthogonal. Also, $m_{1}<\cdots<m_{n}$, and no two of them are $(\omega-N)$-related.

Choose a positive integer $k$ such that

$$
\frac{\omega_{m_{1}+k N}}{\omega_{m_{1}}} \neq \frac{\omega_{m_{n}+k N}}{\omega_{m_{n}}}
$$

A calculation using Lemma 3 shows that

$$
\frac{\omega_{m_{n}+k N}}{\omega_{m_{n}}} p-\left(S^{k}\right)^{*} S^{k}(p)=\sum_{i=1}^{n-1}\left(\frac{\omega_{m_{n}+k N}}{\omega_{m_{n}}}-\frac{\omega_{m_{i}+k N}}{\omega_{m_{i}}}\right) p_{i} .
$$

Since $X$ is reducing for $S$, the above polynomial is still in $X$. The coefficient of $p_{1}$ above is nonzero. If some of the coefficients of $p_{2}, \cdots, p_{n-1}$ are nonzero, we then continue this process. After at most $n-1$ steps, we will have a single term remaining, namely, a nonzero constant multiple of $p_{1}$, which belongs to $X$. Thus $p_{1} \in X$. Similarly, we can show that each $p_{i}$ belongs to $X$.

\section{An EXTREMAL PROBLEM}

For any subspace $X$ of $H_{\omega}^{2}$ let $m=m_{X}$ be the minimal non-negative integer such that there exists some $f \in X$ with $f^{(m)}(0) \neq 0$ but $g^{(k)}(0)=0$ for all $g \in X$ and $0 \leq k \leq m-1$. We will call $m$ the order of zero of $X$ at the origin.

Theorem 6. Let $X$ be a reducing subspace of $S$ in $H_{\omega}^{2}$ with $X \neq\{0\}$ and let $m$ be the order of zero of $X$ at the origin. Then the extremal problem

$$
\sup \left\{\operatorname{Re} f^{(m)}(0): f \in X,\|f\| \leq 1\right\}
$$

has a unique solution $G$ with $\|G\|=1$ and $G^{(m)}(0)>0$. Furthermore, $G$ is a polynomial of degree less than $N$.

Proof. If $f$ is a function in $X$ with Taylor expansion

$$
f(z)=\sum_{k=0}^{\infty} a_{k} z^{k}
$$

then $f^{(m)}(0)=a_{m} m$ !. By the definition of the norm in $H_{\omega}^{2}$, the mapping $f \mapsto$ $f^{(m)}(0)$ is a bounded linear functional on $H_{\omega}^{2}$. It then follows from elementary functional analysis that the extremal problem has a unique solution $G$ with $\|G\|=1$ and $G^{(m)}(0)>0$.

To show that $G$ is a polynomial of degree less than $N$, we consider the functions

$$
g=\frac{G+S f}{\|G+S f\|},
$$

where $f$ is any function in $X$. Since

$$
\operatorname{Re} g^{(m)}(0) \leq G^{(m)}(0),
$$

we obtain

$$
\|G+S f\| \geq 1
$$


for all $f \in X$. This easily implies that $G \perp S X$. In particular, since the function $S^{*} G$ belongs to $X$, we must have $\left\langle S S^{*} G, G\right\rangle=0$, or $S^{*} G=0$. Since the kernel of $S^{*}$ consists of polynomials of degree less than $N$, the proof of the theorem is now complete.

The function $G$ in the theorem above will be called the extremal function of $X$.

Corollary 7. If $X$ is a reducing subspace of $S$ in $H_{\omega}^{2}$, then the order of zero of $X$ at the origin is less than $N$.

Corollary 8. The operator $M_{z}$ is irreducible on $H_{\omega}^{2}$.

Proof. The proof of the theorem above also works when $N=1$. Thus any reducing subspace $X$ of $M_{z}$ in $H_{\omega}^{2}$ with $X \neq\{0\}$ has 0 as the order of zero at the origin; and its associated extremal function must be a nonzero constant. This obviously implies that $X=H_{\omega}^{2}$.

Theorem 9. The extremal function of any reducing subspace of $S$ in $H_{\omega}^{2}$ is transparent.

Proof. Let $G$ be the extremal function of a reducing subspace $X$ of $S$ in $H_{\omega}^{2}$. Let $m$ be the order of zero of $X$ at the origin. If

$$
G=p_{1}+\cdots+p_{n}
$$

is the canonical decomposition of $G$ into transparent polynomials, then $p_{1}$ contains the term $\left(G^{(m)}(0) / m !\right) z^{m}$. Since the $p_{i}$ 's are mutually orthogonal, we have $\left\|p_{1}\right\| \leq$ $\|G\|=1, p_{1}^{(m)}(0)=G^{(m)}(0)$, and $p_{1} \in X$ by Lemma 5 The extremality of $G$ then implies that $G=p_{1}$, and hence $G$ is transparent.

\section{Minimal Reducing SUbSPaCes}

In this section we study minimal reducing subspaces of the operator $S$ in $H_{\omega}^{2}$. Note that in general operators may have reducing subspaces that do not contain minimal reducing subspaces. Actually, there are operators which possess lots of reducing subspaces but have no minimal reducing subspaces at all. Just look at the operator of multiplication by $z$ on the Lebesgue space $L^{2}(\mathbb{D}, d A$ ), where $d A$ is area measure.

Theorem 10. Suppose $X$ is a minimal reducing subspace of $S$ in $H_{\omega}^{2}$. If

$$
p(z)=\sum_{k=m}^{N-1} a_{k} z^{k}
$$

is a polynomial in $X$, where $a_{m} \neq 0$, then $p$ is transparent.

Proof. Assume that there exists some $k$ with $m<k \leq N-1$ such that $a_{k} \neq 0$ and $k$ is not $(\omega-N)$-related to $m$. Then there exists some positive integer $l$ such that

$$
\frac{\omega_{m+l N}}{\omega_{m}} \neq \frac{\omega_{k+l N}}{\omega_{k}}
$$

Consider the function

$$
q=\left(S^{l}\right)^{*} S^{l} p-\frac{\omega_{m+l N}}{\omega_{m}} p .
$$


An easy calculation using Lemma 2 shows that $q$ is a polynomial of the form

$$
q(z)=\sum_{i=m+1}^{N-1} b_{i} z^{i} .
$$

Since $k$ and $m$ are not $(\omega-N)$-related, we must have $b_{k} \neq 0$, so that $q$ is nonzero. The reducing subspace $X_{q}$ is contained in $X$, and every function in $X_{q}$ is missing $z^{m}$ in its Taylor expansion. In particular, $p$ does not belong to $X_{q}$. Thus $X_{q}$ is a nonzero reducing subspace of $S$ that is properly contained in $X$. This contradicts the assumption that $X$ is minimal.

Theorem 11. Let $X$ be a reducing subspace of $S$ in $H_{\omega}^{2}$. Then $X$ is minimal if and only if there is a transparent polynomial $p$ such that

$$
X=X_{p}=\operatorname{Span}\left\{p z^{k N}: k=0,1,2, \cdots\right\} .
$$

Proof. If $X$ is a minimal reducing subspace and $G$ is its associated extremal function, then $G$ is transparent according to Theorem 9 Since $X_{G} \subset X$, the minimality of $X$ together with Lemma 4 gives

$$
X=X_{G}=\operatorname{Span}\left\{p z^{k N}: k=0,1,2, \cdots\right\} .
$$

Next assume that $p$ is a transparent polynomial. To show that the reducing subspace $X_{p}$ is minimal, we assume that $Y$ is another nonzero reducing subspace of $S$ contained in $X_{p}$. Let $G_{Y}$ be the extremal function of $Y$. Then $G_{Y}=p f\left(z^{N}\right)$ for some analytic function $f$. On the other hand, $G_{Y}$ is a polynomial of degree less than $N$. It follows that $f$ must be constant, or $G_{Y}$ is a constant multiple of $p$. In particular, $p \in Y$. The definition of $X_{p}$ then implies that $X_{p} \subset Y$. This shows that $X_{p}=Y$ and hence $X_{p}$ is minimal.

Corollary 12. Every reducing subspace of $S$ in $H_{\omega}^{2}$ contains a minimal reducing subspace.

Proof. If $X$ is a reducing subspace of $S$ in $H_{\omega}^{2}$, then Theorem 9 tells us that the extremal function $G$ of $X$ is transparent. By Theorem 11 the reducing subspace generated by $G$ is minimal and is contained in $X$.

Theorem 13. Every reducing subspace of $S$ in $H_{\omega}^{2}$ is a direct (orthogonal) sum of no more than $N$ minimal reducing subspaces.

Proof. Let $X$ be a nonzero reducing subspace of $S$ in $H_{\omega}^{2}$. Let $G$ be the extremal function of $X$. Then $G$ is transparent and hence $X$ contains the minimal reducing subspace

$$
X_{G}=\operatorname{Span}\left\{G z^{k N}: k=0,1,2, \cdots\right\} .
$$

Let $Y=X \ominus X_{G}$ and continue this process (note that the order of zero of $Y$ at the origin is strictly greater than the order of zero of $X$ at the origin). By Corollary 7 this process will stop in no more than $N$ steps.

\section{Type I Weights}

In this section we give a complete description of the reducing subspaces of $S$ in $H_{\omega}^{2}$ for type I weights. 
Theorem 14. If $\omega$ is of type I, then $X_{n}, 0 \leq n \leq N-1$, are the only minimal reducing subspaces of $S$ in $H_{\omega}^{2}$. There are exactly $2^{N}-2$ proper reducing subspaces of $S$ in $H_{\omega}^{2}$; and they are simply the direct (orthogonal) partial sums of these $X_{n}$ 's.

Proof. If $\omega$ is of type I, then the only transparent polynomials are the monomials of degree less than $N$. The desired results then follow from Theorems 11 and 13

Corollary 15. If $\omega$ is of type I, then the extremal function of every nonzero reducing subspace of $S$ in $H_{\omega}^{2}$ is a monomial.

\section{TYPE II WEIGHTS}

We characterize the reducing subspaces of $S$ in $H_{\omega}^{2}$ for type II weights in this section.

Theorem 16. Suppose $\omega$ is of type II. Then there are infinitely many minimal reducing subspaces of $S$ in $H_{\omega}^{2}$; every minimal reducing subspace is generated by a transparent polynomial; and every reducing subspace is the direct (orthogonal) sum of no more than $N$ minimal reducing subspaces.

Proof. Choose two different integers $0 \leq n \leq N-1$ and $0 \leq m \leq N-1$ such that they are $(\omega-N)$-related. For any complex numbers $a$ and $b$ let

$$
p(z)=a z^{n}+b z^{m}
$$

Then $p$ is transparent and $X_{p}$ is a minimal reducing subspace of $S$ in $H_{\omega}^{2}$. Also, if $p$ and $q$ are two nonzero such polynomials, then $X_{p}=X_{q}$ if and only if the coefficient vectors of $p$ and $q$ are linearly dependent. This shows that there are infinitely many distinct minimal reducing subspaces of the form $X_{p}$. The other two claims have already been proved in earlier sections.

\section{SOME EXAMPLES AND REMARKS}

If $\alpha$ is any nonzero real number, then

$$
\omega_{n}=(n+1)^{\alpha}, \quad n=0,1,2, \cdots,
$$

defines a weight sequence of type I for any $N>1$. Hilbert spaces generated by such weights include the Bergman space and the Dirichlet space.

If $M$ is any positive constant, then

$$
\omega_{n}=M^{n}, \quad n=0,1,2, \cdots,
$$

defines a weight sequence of type II for any $N>1$. Hilbert spaces generated by such weights are the Hardy spaces of disks centered at the origin.

Finally we remind the reader that whether or not a weight sequence is of type I or type II depends on the integer $N$ in the definition of $S=M_{z}^{N}$. However, if $\omega$ is of type I for some $N$, then it is also of type I for all smaller $N$ 's; and if $\omega$ is of type II for some $N$, then it is also of type II for all larger $N$ 's.

\section{REFERENCES}

[1] M.B. Abrahamse and J.A. Ball, Analytic Toeplitz operators with automorphic symbol II, Proc. Amer. Math. Soc. 59 (1976), 323-328. MR 56:12962

[2] J.A. Ball, Hardy space expectation operators and reducing subspaces, Proc. Amer. Math. Soc. 47 (1975), 351-357. MR 50:10887

[3] P. Halmos, Shifts on Hilbert spaces, J. Reine Angew. Math. 208 (1961), 102-112. MR 27:2868 
[4] E. Nordgren, Reducing subspaces of analytic Toeplitz operators, Duke Math. J. 34 (1967), 175-181. MR 35:7155

[5] D. Sarason, Invariant subspaces, Topics in Operator Theory, Mathematical Surveys 13 (1974), 1-47. MR 50:10862

[6] A.L.Shields, Weighted shift operators and analytic function theory, Topics in Operator Theory, Mathematical Surveys 13 (1974), 49-128. MR 50:14341

[7] K. Zhu, Reducing subspaces for a class of multiplication operators, J. London Math. Soc. (2) 62 (2000), 553-568. CMP 2001:01

Department of Mathematics, State University of New York, Albany, New York 12222

E-mail address: stessin@math.albany.edu

Department of Mathematics, State University of New York, Albany, New York 12222

E-mail address: kzhu@math.albany.edu 